\title{
Metal abundances of field A and Am stars ${ }^{\star}$
}

\author{
A. Hui-Bon-Hoa \\ 1 Laboratoire d'Astrophysique Extragalactique et de Cosmologie, CNRS (UMR 8631), Observatoire de Paris, Université Paris 7 , \\ DAEC, Observatoire de Meudon, F-92195 Meudon Cedex, France \\ 2 Département de physique, Université de Moncton, Moncton, N.-B., E1A 3E9, Canada
}

Received April 12, 1999; accepted February 25, 2000

\begin{abstract}
Detailed abundances of 9 field A and Am stars are derived for $\mathrm{Mg}, \mathrm{Ca}, \mathrm{Sc}, \mathrm{Cr}, \mathrm{Fe}$, and Ni using high resolution spectroscopy. Most of our sample Am stars show the characteristic deficiencies of $\mathrm{Ca}$ and Sc on one hand, and the overabundances of the iron peak elements on the other, which is consistent with the predictions of the diffusion model for Main Sequence or slightly evolved stars. There seems to be a correlation between abundances of $\mathrm{Ca}$ and Sc and the effective temperature for stars of similar age: the abundance of $\mathrm{Ca}$ increases with increasing mass whereas that of Sc decreases. Two of our Am stars have a different abundance pattern: $\mathrm{Ca}$ is overabundant in the hot magnetic Am star o Peg and its abundance is nearly normal in the metallic giant star $\rho$ Pup. The other objects of our sample have globally a solar composition except 28 And, which shows significant deficiencies for most the studied elements.
\end{abstract}

Key words: stars: abundances; chemically peculiar

\section{Introduction}

A significant number of upper Main Sequence stars show spectral peculiarities, which reflect abundance anomalies (Preston 1974). Microscopic diffusion is considered to be the main physical process that creates these anomalies by the building of an abundance stratification in the stable layers of the star (Michaud 1970; Michaud et al. 1976). The stratification and the abundances vary with time (Michaud \& Charland 1986; Alecian 1986, 1996). Among the Chemically Peculiar stars, most studies on abundance variation due to diffusion concern Am stars (see Alecian

\footnotetext{
Send offprint requests to: A. Hui-Bon-Hoa

* Based on observations collected at the Observatoire de Haute-Provence (France), and on data from the ESA HIPPARCOS astrometry satellite.
}

1996; Richer et al. 2000). However, the simplest diffusion models lead to anomalies much larger than observed (Michaud et al. 1976). More sophisticated models, which include competing processes, are needed. The abundance evolution of $\mathrm{Ca}$ (and Sc but with less reliable atomic data) was investigated by Alecian (1996) taking into account mass-loss and an extension of the superficial mixing zone below the convective zone. The abundance evolution with time is very sensitive to these competing processes. A phase of overabundance may occur for very young stars. Richer et al. (2000) simulated the evolution of Am stars considering consistently the radiative diffusion of 28 elements and isotopes, and turbulence. An important result is that the strength of the abundance anomalies depends strongly on the amount of material affected by the turbulent mixing.

To constrain these models, abundance analyses of stars with various ages are needed: cluster stars or field stars with a precise location in the $\mathrm{H}-\mathrm{R}$ diagram. Hence, we present a study of field stars belonging to the HIPPARCOS sample. The abundance analysis is performed using the same instrument and method as for the cluster stars of Hui-Bon-Hoa and coworkers and completes these previous works towards the end of the Main Sequence lifetime.

\section{Observations and reductions}

Our 9 field A and Am stars are bright, slow rotating, and (mostly) slightly evolved objects still located in or very near the Main Sequence strip (Burkhart \& Coupry 1991; HIPPARCOS, ESA 1997). Several objects are of special interest: $\rho$ Pup and $\tau$ UMa are metallic-line giants (Gray \& Garrison 1989); RR Lyn is a metallic-line SB2 system (Popper 1971); o Peg is a hot Am star with a magnetic field (Mathys \& Lanz 1990).

The observing run was in December 1996 with the AURELIE spectrograph (Gillet et al. 1994) at the coudé focus of the $152 \mathrm{~cm}$ telescope of the Observatoire de 
Table 1. Basic data for the programme stars

\begin{tabular}{ccccc}
\hline Name & HD & Sp. type & $V$ mag & Remarks \\
\hline 28 And & 2628 & A7III (1) & 5.23 & $\delta$ Scuti, $p=0.07 \mathrm{~d}$ \\
9 Aur & 32537 & F0V (1) & 5.00 & SBO, $p=392 \mathrm{~d}$ \\
14 Aur & 33959 & A9IV $\delta$ Del (1) & 5.02 & SBO, $p=3.79 \mathrm{~d} ; \delta$ Scuti, $p=0.09 \mathrm{~d}$ \\
6 Mon & 43760 & F0 $\delta$ Del $(1)$ & 6.75 & Var Vr? \\
RR Lyn & 44691 & A3Vm (1) & 5.64 & SB2O, $p=9.95 \mathrm{~d}$ \\
$\rho$ Pup & 67523 & F6If $\delta$ Del (1) & 2.81 & kF3hF5mF5(Ib-II) $(2) ; \delta$ Scuti, $p=0.14 \mathrm{~d}$ \\
$\tau$ UMa & 78362 & Am (1) & 4.67 & SBO, $p=1062 \mathrm{~d}$ \\
32 Aqr & 209625 & A5m (1) & 5.30 & SB1O, $p=7.83 \mathrm{~d}$ \\
$o$ Peg & 214994 & A1IV (1) & 4.79 & Am (3); Magnetic field $(4)$ \\
\hline
\end{tabular}

References: (1) Hoffleit (1982); (2) Gray \& Garrison (1989); (3) Conti \& Strom (1968); (4) Mathys \& Lanz (1990).

Haute-Provence. The settings are the same as those of Hui-Bon-Hoa et al. (1997, hereafter HBA97), giving a spectral resolution of about 34000 (linear dispersion of $5 \AA \mathrm{mm}^{-1}$ ) and a spectral interval 5495 - 5620 $\mathrm{A}$ (typical spectra are shown in Fig. 1 of HBA97). The detector was a linear diode array Thomson TH 7832 with 203613 by $750 \mu \mathrm{m}$ pixels. The integration time varied from $10 \mathrm{~min}-$ utes to 2 hours to have signal-to-noise ratios of at least 300. Offset level measurements, exposures of a tungsten lamp and of a thorium-argon hollow cathode lamp were regularly performed for calibration purpose. Codes written by M. Spite (1967, 1996 private communication) were used to reduce the spectra (see HBA97).

\section{Analysis}

We used the uvby $\beta$ photometry to estimate effective temperatures and gravities (Moon \& Dworetsky 1985; Moon 1985; Napiwotzki et al. 1993). The uncertainties in the photometric measurements are the major sources of the errors: $\pm 200 \mathrm{~K}$ for $T_{\text {eff }}$ and \pm 0.14 for $\log g$. A variation of $200 \mathrm{~K}$ in $T_{\text {eff }}$ induces a change of 0.07 dex in the abundances, whereas a variation of 0.3 dex in $\log g$ (twice the error) induces a change of 0.1 dex for the singly ionized species, the neutrals being almost unchanged. The compilation of Hauck \& Mermilliod (1980) provided the photometric data, which are dereddened using the code of Moon (1985). The fundamental parameters of the SB2 system RR Lyn are determined using the data of Popper (1971). The error in $T_{\text {eff }}$ is $\pm 400 \mathrm{~K}$, yielding uncertainties of 0.1 (singly ionized species) and $0.2 \mathrm{dex}$ (neutrals) in the abundances.

The abundance analysis is carried out assuming LTE with the codes of M. Spite (1967, 1996 private communication). We used Kurucz' ATLAS9 code (Kurucz 1993) to build the model atmospheres, the overshooting option being switched off since models computed this way are generally in better agreement with observations (van't Veer \& Mégessier 1996; Castelli et al. 1997). The treatment of convection (mixing length theory or Canuto \& Mazzitelli 1991, 1992) and the microturbulent velocity of the model have negligible influence on the abundances derived with the present set of lines (Hui-Bon-Hoa 1998, 1999). For most sample stars, the microturbulent velocity $\left(V_{\mathrm{t}}\right)$ minimizes the dispersion among the abundance values from the different lines of FeI. The value for $o$ Peg (which could correspond to a pseudo-microturbulence due to the magnetic field, Adelman 1973) is chosen following recent abundance studies (Adelman et al. 1984; Adelman 1988a,b; Hill \& Landstreet 1993). That of the fainter component of RR Lyn uses the relationship between $V_{\mathrm{t}}$ and $T_{\text {eff }}$ of Coupry \& Burkhart (1992).

The lines used for the abundance determination and their equivalent widths are listed in Table 2. For each component of RR Lyn, the dilution factors of Popper (1971) are applied (1.5 for the brighter star and 3.0 for the other). We used scaled solar $g f$-values (Col. 3 of Table 2), obtained by fitting the solar spectrum with the Kurucz solar model, the abundances of Grevesse et al. (1996) and a microturbulent velocity of $1 \mathrm{~km} \mathrm{~s}^{-1}$. The equivalent width measurements are carried out with the method of Cayrel et al. (1985) and the smallest values used are equal to the systematic error made on the equivalent widths, estimated using the expression of Cayrel (1988). The hyperfine structure of Sc is neglected.

\section{Results}

Our abundance results and the fundamental stellar parameters are listed in Table 3 . The abundance patterns are shown in Fig. 1 for the stars we classified as Am and in Fig. 2 for the other objects. Comparisons with other studies are presented in Table 4. The solar abundances are those of Grevesse et al. (1996): Mg, 7.58; Ca, 6.36; Sc, 3.17; Cr, 5.67; Fe, 7.50; Ni, 6.25. The script $[X]$ for any quantity $X$ means $\log (X)_{*}-\log (X)_{\odot}$. The dispersion of the values given by different lines is indicated for $\mathrm{Ca}$ and $\mathrm{Fe}$ when more than two lines are used for the estimate. The $v \sin i$ values are deduced from the width of the gaussian fit used for the equivalent width measurements (Hui-Bon-Hoa \& Alecian 1998).

A star is classified as an Am star when its spectral type given by the CaII-K line is earlier than that derived 
Table 2. Equivalent widths in $\mathrm{m} \AA$ for the sample stars (HD numbers). The two sets of values for HD 78362 correspond the two spectra we took

\begin{tabular}{|c|c|c|c|c|c|c|c|c|c|c|c|c|c|}
\hline$\lambda(\AA)$ & Element & $\log g f$ & 2628 & 32537 & 33959 & 43760 & $44691 \mathrm{~A}$ & 44691B & 67523 & $78362(1)$ & $78362(2)$ & 209625 & 214994 \\
\hline 5501.47 & FeI & -2.95 & 48 & - & 44 & - & 61 & - & 146 & 118 & 120 & 58 & 4 \\
\hline 5502.09 & CrII & -1.92 & 31 & 28 & 35 & 47 & - & 22 & 88 & 99 & 100 & 54 & 20 \\
\hline 5506.79 & FeI & -2.80 & 67 & 72 & 58 & 90 & 92 & - & 172 & 133 & 134 & 79 & - \\
\hline 5508.64 & CrII & -1.98 & 20 & 21 & 27 & 35 & 54 & 23 & 75 & 78 & 81 & 43 & 15 \\
\hline 5512.99 & CaI & -0.29 & - & - & 38 & 50 & 15 & 57 & - & - & - & 16 & - \\
\hline 5526.82 & ScII & 0.08 & 97 & 84 & 98 & 126 & 8 & 58 & 133 & 61 & 59 & 18 & 14 \\
\hline 5528.42 & MgI & -0.62 & 160 & 143 & 148 & 144 & 167 & 95 & 230 & 179 & 179 & 147 & 37 \\
\hline 5543.20 & $\mathrm{FeI}$ & -1.57 & - & 30 & - & 35 & 38 & 23 & 85 & 72 & 71 & 33 & - \\
\hline 5543.95 & FeI & -1.09 & - & 31 & - & 37 & 50 & 23 & 82 & 72 & 70 & 33 & - \\
\hline 5554.90 & FeI & -0.32 & 44 & 48 & 40 & 59 & 69 & - & 111 & 103 & 102 & 60 & 12 \\
\hline 5560.22 & FeI & -1.19 & - & 19 & 12 & 20 & 27 & 24 & - & 53 & 50 & 22 & - \\
\hline 5569.63 & FeI & -0.54 & 86 & 87 & 74 & 98 & 124 & 58 & 175 & 158 & 154 & 108 & 16 \\
\hline 5576.10 & FeI & -0.92 & 62 & 67 & 53 & - & 84 & - & 146 & 128 & 126 & 72 & 8 \\
\hline 5578.73 & $\mathrm{NiI}$ & -2.65 & 7 & 8 & - & 16 & 16 & 20 & 39 & 50 & 47 & 15 & 3 \\
\hline 5581.98 & CaI & -0.71 & 53 & 60 & 48 & 62 & 22 & 41 & 98 & 30 & 28 & 24 & - \\
\hline 5586.77 & FeI & -0.10 & 125 & 117 & 111 & 138 & - & 80 & 222 & 206 & 205 & 160 & 31 \\
\hline 5588.77 & CaI & 0.21 & 128 & 121 & 117 & - & 78 & - & 189 & 99 & 94 & 86 & 11 \\
\hline 5589.37 & NiI & -1.14 & 5 & - & 3 & 14 & 9 & 25 & 31 & 43 & 43 & 16 & 2 \\
\hline 5590.13 & CaI & -0.71 & 50 & 60 & 43 & 56 & 23 & 44 & 94 & 29 & 29 & 22 & - \\
\hline 5593.75 & NiI & -0.83 & 10 & 12 & 9 & 22 & - & 15 & 48 & 64 & 62 & 26 & 2 \\
\hline 5601.29 & $\mathrm{CaI}$ & -0.35 & 62 & 77 & 55 & 73 & 38 & - & 128 & 70 & 66 & 37 & - \\
\hline
\end{tabular}

Table 3. Abundance values for the programme stars. A colon (:) denote uncertain values (see text)

\begin{tabular}{|c|c|c|c|c|c|c|c|c|c|c|c|c|c|}
\hline Name & HD & Remarks & $\begin{array}{c}v \sin i \\
\left(\mathrm{~km} \mathrm{~s}^{-1}\right)\end{array}$ & $\begin{array}{l}T_{\text {eff }} \\
(\mathrm{K})\end{array}$ & $\log g$ & $\begin{array}{c}V_{\mathrm{t}} \\
\left(\mathrm{km} \mathrm{s}^{-1}\right)\end{array}$ & {$[\mathrm{Mg} / \mathrm{H}]$} & {$[\mathrm{Ca} / \mathrm{H}]$} & {$[\mathrm{Sc} / \mathrm{H}]$} & {$[\mathrm{Cr} / \mathrm{H}]$} & {$[\mathrm{Fe} / \mathrm{H}]$} & {$[\mathrm{Ni} / \mathrm{H}]$} & Type \\
\hline 28 And & 2628 & $\delta$ Scuti & 21 & 7270 & 3.5 & 3.0 & -0.08 & $\begin{array}{c}-0.26 \\
( \pm 0.12)\end{array}$ & -0.41 & -0.38 & $\begin{array}{c}-0.40 \\
( \pm 0.06)\end{array}$ & -0.19 & atyp \\
\hline 9 Aur & 32537 & $\mathrm{SBO}$ & 21 & 7030 & 4.1 & 1.5 & -0.02 & $\begin{array}{c}-0.03 \\
( \pm 0.09)\end{array}$ & -0.06 & -0.19 & $\begin{array}{c}-0.13 \\
( \pm 0.07)\end{array}$ & -0.35 & $\mathrm{nl}$ \\
\hline 14 Aur & 33959 & $\mathrm{SBO} ; \delta$ Scuti & 26 & 7670 & 3.5 & 2.5 & 0.16 & $\begin{array}{c}-0.03 \\
( \pm 0.15)\end{array}$ & -0.16 & -0.19 & $\begin{array}{c}-0.14 \\
( \pm 0.10)\end{array}$ & -0.09 & $\mathrm{nl}$ \\
\hline 6 Mon & 43760 & Var Vr? & 13 & 7170 & 3.2 & 2.5 & -0.14 & $\begin{array}{c}-0.15 \\
( \pm 0.12)\end{array}$ & -0.03 & -0.20 & $\begin{array}{c}-0.05 \\
( \pm 0.11)\end{array}$ & 0.17 & $\mathrm{nl}$ \\
\hline RR Lyn A & $44691 \mathrm{~A}$ & $\mathrm{~A} 3 \mathrm{Vm}(1) ; \mathrm{SB} 2 \mathrm{O}$ & 18 & 8240 & 4.0 & 3.0 & 0.52 & $\begin{array}{c}-0.13 \\
( \pm 0.13)\end{array}$ & -1.38 & 0.43 & $\begin{array}{c}0.66 \\
( \pm 0.12)\end{array}$ & 0.76 & $\mathrm{Am}$ \\
\hline RR Lyn B & $44691 \mathrm{~B}$ & & 16 & 7610 & 4.1 & 2.5: & -0.66 : & 0.07: & $-0.52:$ & -0.20 : & $-0.19:$ & $0.46:$ & $\mathrm{Am}$ \\
\hline$\rho$ Pup & 67523 & kF3hF5mF5(Ib-II) $(2) ; \delta$ Scuti & 17 & 6920 & 3.7 & 3.5 & 0.31 & $\begin{array}{c}0.14 \\
( \pm 0.07)\end{array}$ & -0.17 & 0.38 & $\begin{array}{c}0.36 \\
( \pm 0.10)\end{array}$ & 0.40 & $\mathrm{Am}$ \\
\hline$\tau \mathrm{UMa}$ & 78362 & $\operatorname{Am}(1) ; \mathrm{SBO}$ & 13 & 7390 & 4.2 & 3.0 & 0.14 & $\begin{array}{c}-0.42 \\
( \pm 0.10)\end{array}$ & -0.56 & 0.73 & $\begin{array}{c}0.56 \\
( \pm 0.07)\end{array}$ & 0.85 & $\mathrm{Am}$ \\
\hline $32 \mathrm{Aqr}$ & 209625 & $\operatorname{A} 5 \mathrm{~m}(1) ; \mathrm{SB} 1 \mathrm{O}$ & 10 & 7870 & 3.9 & 3.2 & -0.02 & $\begin{array}{c}-0.36 \\
( \pm 0.13)\end{array}$ & -1.15 & 0.20 & $\begin{array}{c}0.26 \\
( \pm 0.12)\end{array}$ & 0.62 & $\mathrm{Am}$ \\
\hline$o$ Peg & 214994 & Am (3); Magnetic field (4) & $\leq 10$ & 9650 & 3.6 & 1.5 & 0.07 & 0.32 & -0.35 & 0.19 & $\begin{array}{c}0.42 \\
( \pm 0.17)\end{array}$ & 1.13 & Am \\
\hline
\end{tabular}

References: (1) Hoffleit (1982); (2) Gray \& Garrison (1989); (3) Conti \& Strom (1968); (4) Mathys \& Lanz (1990).

from the metallic lines, the hydrogen line type being inbetween (Roman et al. 1948). This reflects the following abundance anomalies: deficiency of $\mathrm{Ca}$ and/or overabundance of heavy elements (Conti 1970). The scandium deficiency is at least as typical in Am stars and is considered as a complementary criterion (see Conti 1970 and references therein). Thus, we classified our sample stars as Am stars when $\mathrm{Ca}$ and/or Sc are deficient and/or at least two heavy elements are overabundant, whereas normal A stars are those which exhibit normal abundances for $\mathrm{Ca}, \mathrm{Sc}$, and at least for two of the elements $\mathrm{Cr}, \mathrm{Fe}$, or Ni (abundances are considered as normal when within a factor 2 around the solar value, i.e. $\pm 0.3 \mathrm{dex})$. Stars that do not belong to the previous groups are denoted "atypical". This clas- sification is shown in the last column of Table 3 (resp. by the notation Am, nl, and atyp).

\subsection{Am stars}

Most Am stars show more or less the classical Am abundance pattern. However, some objects deviate obviously from this picture.

RR Lyn (HD 44691) is an SB2 Am system whose brighter component (labelled A) has a classical pattern but that of the companion is very different: Sc is much less deficient and $\mathrm{Ni}$ is the only overabundant iron peak element. 


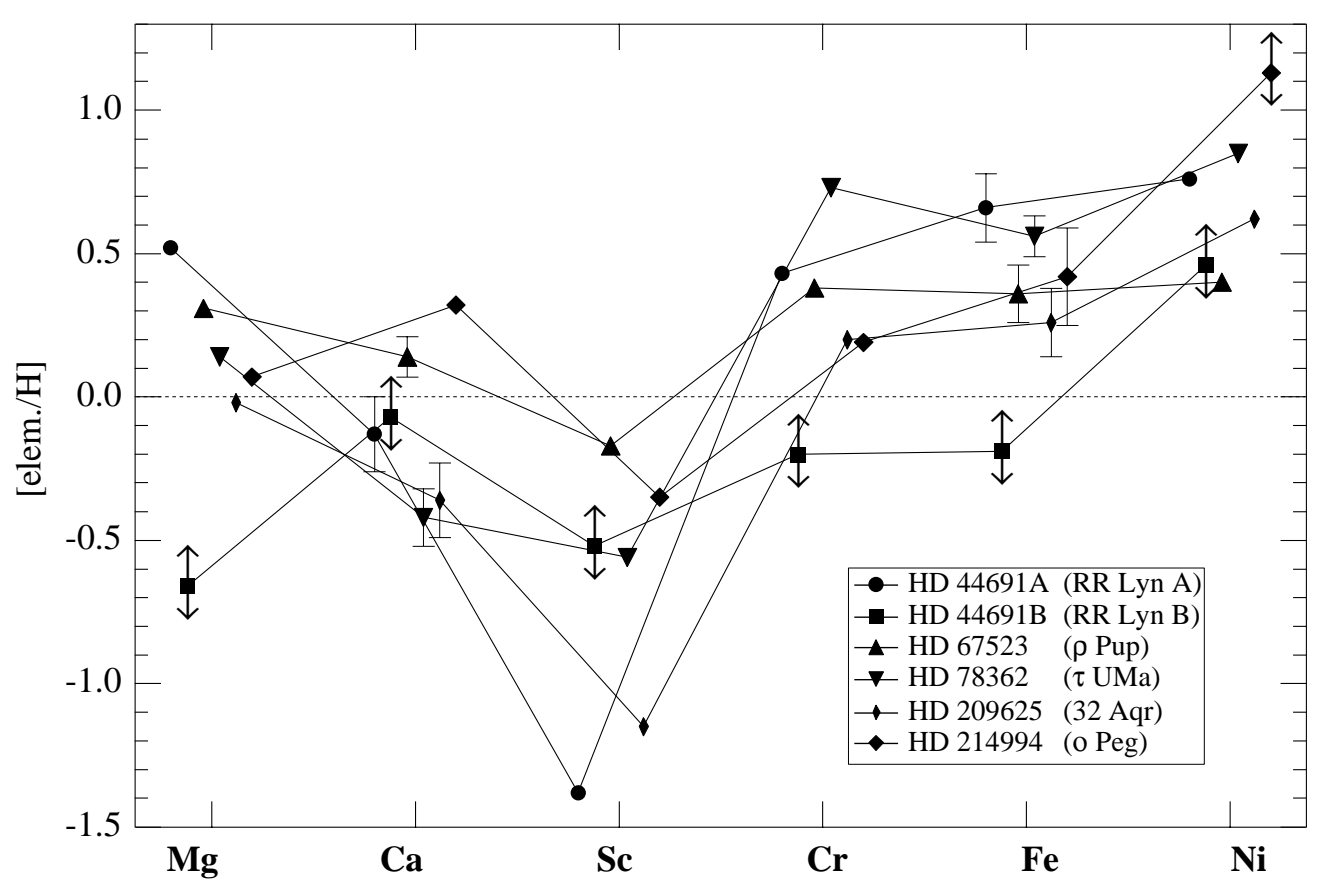

Fig. 1. Abundance patterns for Am stars. Error bars ended by arrows denote very uncertain values (see text for details)

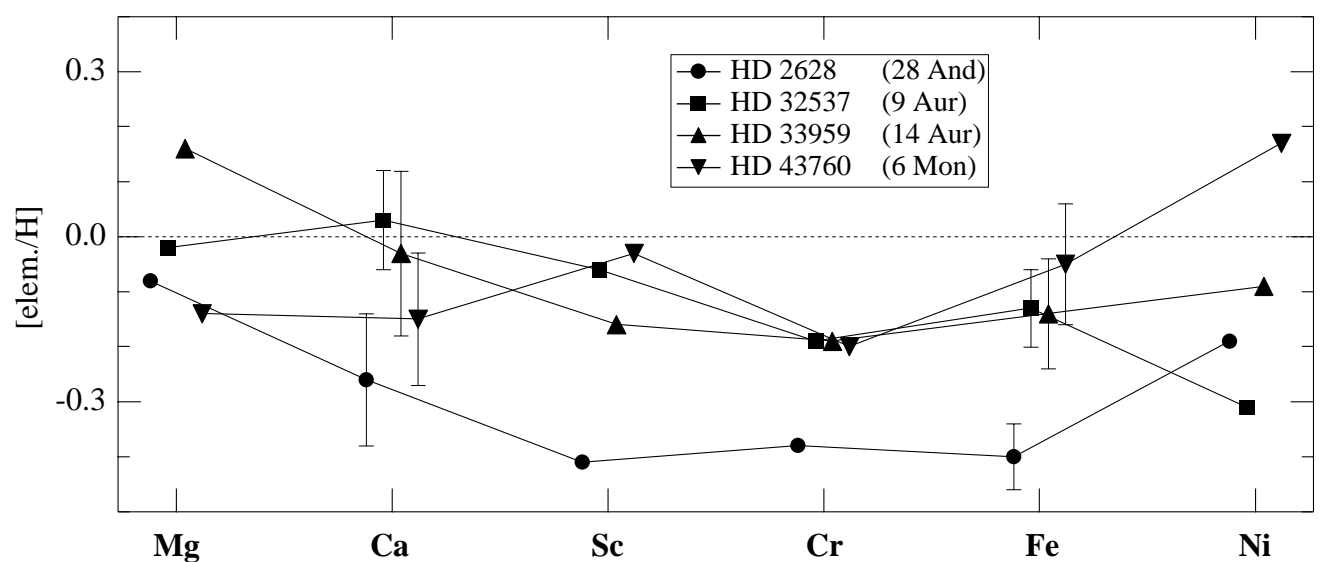

Fig. 2. Same as Fig. 1 for non-Am stars

$\rho$ Pup (HD 67523) is the prototype of an eponymous group of evolved metallic-line stars (Gray \& Garrison 1989). The iron peak elements are enhanced, Ca slightly overabundant and Sc mildly deficient, yielding a pattern of Conti's (1970) type c, i.e. star with only overabundances of heavy elements.

$o$ Peg (HD 214992) is a hot Am star (Conti \& Strom 1968 ) in which a magnetic field of about $2 \mathrm{kG}$, with a complex structure, has been detected (Mathys 1988; Mathys \& Lanz 1990). Our results agree with those of previous studies (see Adelman et al. 1984; Adelman 1988a,b; Hill \& Landstreet 1993) but, with almost same atmospheric parameters, our calcium abundance is clearly higher. This may be due to non-LTE effects or reflect an abundance stratification: the Car lines of Adelman and coworkers have lower excitation potential as ours and may form differently; the results of Hill \& Landstreet are obtained by the simultaneous adjustment of both neutral and singly ionized Ca lines and should be considered as values averaged over two ionization stages. With the equivalent widths of 


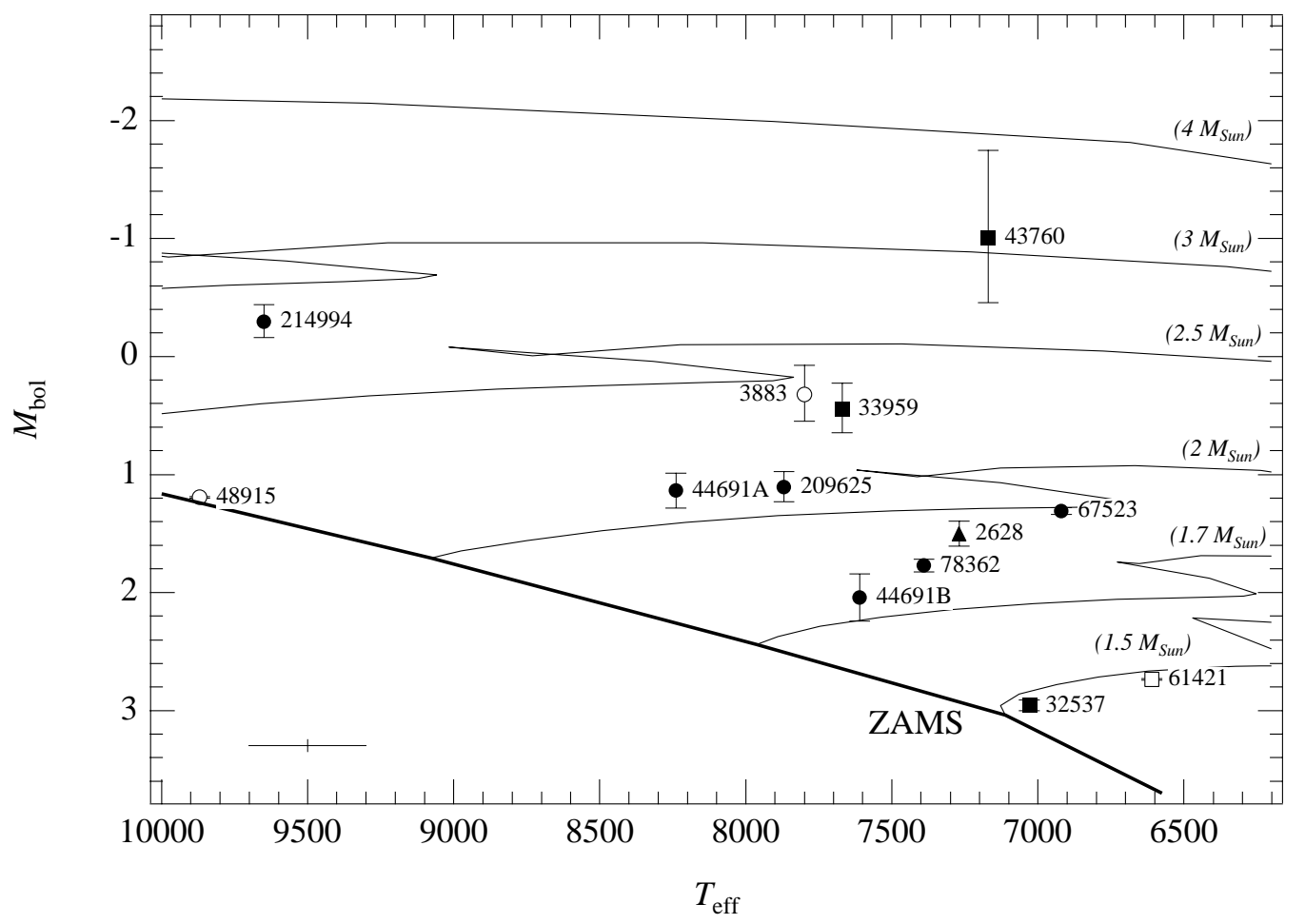

Fig. 3. $\mathrm{H}-\mathrm{R}$ diagram for the programme stars. Dots (and circles) denote Am stars, squares normal stars, and triangles atypical ones. Open symbols refer to objects studied in Hui-Bon-Hoa et al. (1997). Evolutionary tracks of Schaller et al. (1992) are shown with the corresponding stellar masses in parenthesis. The typical uncertainty on $T_{\text {eff }}$ is represented in the lower-left corner. The error bars on $M_{\mathrm{bol}}$ correspond to the standard error on the parallax

Table 4. Comparisons with previous studies

\begin{tabular}{ccc}
\hline HD 209625 & Adelman et al. (1997) & This study \\
\hline$[\mathrm{Mg} / \mathrm{H}]$ & -0.51 & -0.02 \\
{$[\mathrm{Ca} / \mathrm{H}]$} & -0.47 & -0.36 \\
{$[\mathrm{Sc} / \mathrm{H}]$} & -1.16 & -1.15 \\
{$[\mathrm{Cr} / \mathrm{H}]$} & 0.23 & 0.20 \\
{$[\mathrm{Fe} / \mathrm{H}]$} & 0.05 & 0.26 \\
{$[\mathrm{Ni} / \mathrm{H}]$} & 0.53 & 0.62 \\
$T_{\text {eff }}$ & 7700 & 7870 \\
$\log g$ & 3.65 & 3.9 \\
$V_{\mathrm{t}}$ & 4.5 & 3.2 \\
\hline \multicolumn{3}{c}{} \\
\hline $\mathrm{HD} 214994$ & Adelman $(1988 \mathrm{~b})$ & This study \\
\hline$[\mathrm{Mg} / \mathrm{H}]$ & -0.07 & 0.07 \\
{$[\mathrm{Ca} / \mathrm{H}]$} & 0.03 & 0.32 \\
{$[\mathrm{Sc} / \mathrm{H}]$} & -0.47 & -0.35 \\
{$[\mathrm{Cr} / \mathrm{H}]$} & 0.16 & 0.19 \\
{$[\mathrm{Fe} / \mathrm{H}]$} & 0.18 & 0.42 \\
{$[\mathrm{Ni} / \mathrm{H}]$} & 0.44 & 1.13 \\
$T_{\text {eff }}$ & 9600 & 9650 \\
$\log g$ & 3.6 & 3.6 \\
$V_{\mathrm{t}} g$ & 1.8 & 1.5 \\
\hline
\end{tabular}

Conti \& Strom (1968) and our method, we find the same trend of higher values with CaI lines of higher excitation potential ( $g f$-values of Wiese et al. 1969). The strong $\mathrm{Ni}$ overabundance should be considered carefully because of the weakness of the lines.

\subsection{Other stars}

28 And (HD 2628) shows deficiencies for all the studied elements. Here, Sc, Cr, and Fe are obviously underabundant, by an amount comparable to that found in Vega (Adelman \& Gulliver 1990) for the two last elements. A study including more elements would be interesting for a more thorough comparison. The remaining sample stars are almost normal. There is however a trend to slight underabundances for all elements. The values are very close to each other for $\mathrm{Cr}$ and $\mathrm{Fe}$.

\section{Discussion}

Our sample mostly consists of nearby stars (less than $100 \mathrm{pc}$ far) for which we can estimate the absolute magnitude with HIPPARCOS parallaxes (ESA 1997) and rather small uncertainties. Figure 3 shows their location in the $\mathrm{H}-\mathrm{R}$ diagram (the data for $\mathrm{RR}$ Lyn are from Popper 1971). The corrections for absorption (derived from $E(b-y))$ are generally very small (about $0.04 \mathrm{mag}$ ). We do not correct for duplicity since our high $\mathrm{S} / \mathrm{N}$ spectra do not show any secondary spectrum for the SB1 stars, implying that the companion would contribute to less than 0.05 percents to the total luminosity yielding a shift of 
Table 5. Age and mass for the programme stars (including the field stars of HBA97)

\begin{tabular}{cccc}
\hline Name & HD & Mass $\left(M_{\odot}\right)$ & $\log t$ \\
\hline 28 And & 2628 & 1.92 & 9.01 \\
HR 178 & 3883 & 2.44 & 8.78 \\
9 Aur & 32537 & 1.47 & 8.92 \\
14 Aur & 33959 & 2.37 & 8.81 \\
6 Mon & 43760 & 3.14 & 8.51 \\
RR LynA & $44691 \mathrm{~A}$ & 2.10 & 8.83 \\
RR LynB & $44691 \mathrm{~B}$ & 1.77 & 8.94 \\
Sirius & 48915 & 2.21 & $\leq 8.50$ \\
Procyon & 61421 & 1.48 & 9.29 \\
$\rho$ Pup & 67523 & 1.99 & 9.02 \\
$\tau$ UMa & 78362 & 1.84 & 9.02 \\
32 Aqr & 209625 & 2.09 & 8.89 \\
$o$ Peg & 214994 & 2.82 & 8.54 \\
\hline
\end{tabular}

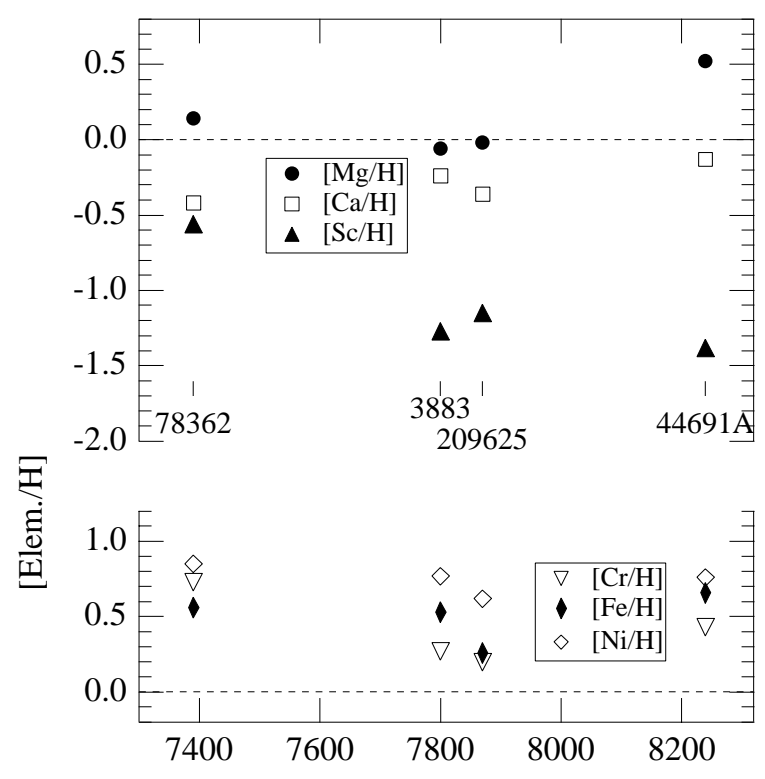

Fig. 4. Abundances against effective temperature for the typical Am stars in the range $7000-8500 \mathrm{~K}$

at most 0.05 mag. We derived the age and mass by interpolation in the evolutionary tracks of Schaller et al. (1992), assuming that the Am stars follow the same tracks as normal solar composition stars of similar mass, as the chemical anomalies of Am stars only affect the superficial layers. The mass can be overestimated for the stars near the upper boundary of the MS (namely HD 3883, 33959, 67523 ) since we assume that they are still in a MS stage though one or even two evolutionary tracks corresponding to lighter and more evolved stars also pass by these points (see Sect. 2.1 of Asiain et al. 1997). The resulting ages and masses are shown in Table 5 . We could only assign an upper limit for Sirius, being very close to the ZAMS.

The typical pattern (underabundances of $\mathrm{Ca}$ and Sc, heavy elements enhanced) is obvious for most Am stars and the anomalies are of comparable strengths as those observed in the oldest cluster stars studied by Hui-BonHoa and coworkers (namely in the Hyades and Praesepe) whose ages are similar to those of our field Am stars (except Sirius). Apart from RR LynB, whose abundances are not reliable enough, two stars show significant differences from this pattern. First, $\rho$ Pup has quasi normal Ca and Sc. Since it is in the very upper part of the Main Sequence strip, the extension of the superficial mixing zone occurring when a star leaves the MS could reduce the strength of the abundance anomalies (Richer et al. 2000). The disappearance of the anomalies occurs sooner for $\mathrm{Cr}$ and $\mathrm{Fe}$ than for Ca, as suggested by the models (Richer 2000, private communication). The $\mathrm{Ca}$ value for $o \mathrm{Peg}$ is more than 0.4 dex enhanced in comparison with that of Sirius. As both have similar atmospheric parameters, this discrepancy may be due to a magnetic field in $o$ Peg. Borsenberger et al. (1981) predict normal abundances of Ca for magnetic Ap stars under the effect of diffusion. A careful study of the stratification would help understand the origin of the dependence of the $\mathrm{Ca}$ abundance on excitation potential (this trend appears because the abundance determination assumes homogeneous content throughout the atmosphere and LTE).

There seems to be a correlation between $[\mathrm{Sc} / \mathrm{H}]$ and $T_{\text {eff }}(r=0.73, \mathrm{~F}(0.05)=23.6)$ in our typical Am stars (Sirius is not included), the underabundances of Sc being more pronounced with increasing temperature (see Fig. 4). An age effect cannot be invoked as our stars are of the same age. This trend is also visible in the oldest cluster Am stars (members of the Hyades and Praesepe, see Fig. 3 of Hui-Bon-Hoa \& Alecian 1998). A reverse tendency is followed by $\mathrm{Ca}$ in the same stars $(r=0.30, \mathrm{~F}(0.05)=3.7)$. Such correlations can be understood considering that the visible abundance is the reflect of that present at the bottom of the superficial convective zone (SCZ), whose location is very sensitive to $T_{\text {eff }}$ (it is located upper in the star for increasing temperature). In the case of $\mathrm{Ca}$, the abundance at the bottom of the SCZ grows as $T_{\text {eff }}$ increases. The radiative accelerations also depend on $T_{\text {eff }}$, but much less sensitively (see Figs. 5 and 6 of Richer et al. 2000) and we can therefore reasonably expect that the shape of the stratification profile is slowly temperature-dependent too at a given age, at least for a limited temperature range (say $1000 \mathrm{~K}$ ). The abundance variation against $T_{\text {eff }}$ would then roughly reflect the stratification profile in the temperature range of interest. In the case of $\mathrm{Ca}$, the local abundance should be larger when one goes to upper layers, which is consistent with the stratification profile predicted by Alecian (1998) and that of Richer et al. (2000) for their 1.6 $M_{\odot}$ model. Unfortunately, they do not consider up to now the case of Sc. Also, we should not forget that effective temperature is not the only parameter on which depends the location of the bottom of the SCZ and this could explain the abundance dispersion at similar $T_{\text {eff }}$ and age. 
As for the normal stars, their quasi-solar patterns may reflect the efficiency of the superficial mixing mechanisms: the He convective should have remained in the MS stars, otherwise they should show obvious abundance anomalies, even the less massive of them (Turcotte et al. 1998). 6 Mon is in a post-MS stage where dredge-up is certainly active.

The pattern of 28 And cannot be safely discussed until a more complete picture of the anomalies is available. However, owing to certain similarities with the anomalies presented by Vega, the diffusion model does not seem to be the relevant one and one should rather consider the models proposed to explain the $\lambda$ Boö phenomenon.

Acknowledgements. I would like to thank G. Alecian for fruitful discussions. The referee S.J. Adelman made many interesting comments that helped to improve this paper. Thanks to the OHP staff who provided an excellent support. This research made use of the bibliographical stellar database, Simbad, operated at the Centre de Données Stellaires (Strasbourg, France).

\section{References}

Adelman S.J., 1973, ApJ 183, 959

Adelman S.J., 1988a, MNRAS 230, 671

Adelman S.J., 1988b, MNRAS 235, 749

Adelman S.J., Gulliver A.F., 1990, ApJ 348, 712

Adelman S.J., Young J.M., Baldwin H.E., 1984, MNRAS 206, 649

Adelman S.J., Caliskan H., Kocer D., Bolcal C., 1997, MNRAS 288,470

Alecian G., 1986, A\&A 168, 204

Alecian G., 1996, A\&A 310, 872

Alecian G., 1998, Contrib. Astron. Obs. Skalnaté Pleso, Vol. XXVII, No. 3 North P., Schnell A., Ziznovsky J. (eds.), p. 290

Asiain R., Torra J., Figueras F., A\&A 322, 147

Borsenberger J., Praderie F., Michaud G., 1981, ApJ 243, 533

Burkhart C., Coupry M.-F., 1991, A\&A 249, 205

Canuto V.M., Mazzitelli I., 1991, ApJ 370, 295

Canuto V.M., Mazzitelli I., 1992, ApJ 389, 724

Castelli F., Gratton R.G., Kurucz R.L., 1997, A\&A 318, 841

Cayrel R., 1988, in: The Impact of very High S/N Spectroscopy on Stellar Physics, IAU Symp. 132, Cayrel de Strobel G. and Spite M. (eds.), p. 345

Cayrel R., Cayrel de Strobel G., Campbell B., 1985, A\&A 146, 249
Conti P.S., 1970, PASP 82, 781

Conti P.S., Strom S.E., 1968, ApJ 154, 975

Coupry M.-F., Burkhart C., 1992, A\&AS 95, 41

ESA 1997, The Hipparcos Catalogue, ESA SP-1200

Gillet D., Burnage R., Kohler D., et al., 1994, A\&AS 108, 181

Gray R.O., Garrison R.F., 1989, ApJS 69, 301

Grevesse N., Noels A., Sauval A.J., 1996, in: Cosmic Abundances, Holt S. and Sonneborn G. (eds.). San Francisco, ASP Conf. Ser. 99, 117

Hauck B., Mermilliod J.C., 1980, A\&AS 40, 1

Hill G.M., Landstreet J.D., 1993, A\&A 276, 142

Hoffleit D., 1982, The Bright Star Catalogue, Yale Univ. Obs., New Haven, Connecticut

Hui-Bon-Hoa A., 1998, Thèse de Doctorat, Université Paris 7

Hui-Bon-Hoa A., 1999, A\&A 343, 261

Hui-Bon-Hoa A., Alecian G., 1998, A\&A 332, 224

Hui-Bon-Hoa A., Burkhart C., Alecian G., 1997, A\&A 323, 901 (HBA97)

Kurucz R.L., 1993, ATLAS9 Stellar Atmosphere Programs and $2 \mathrm{~km} \mathrm{~s}^{-1}$ grid (Kurucz CD-ROM No. 13). Smithsonian Astrophysical Observatory

Mathys G., 1988, in: Elemental Abundance Analyses, Inst. Astr. Univ. Lausanne, Chavannes-des-Bois, Adelman S.J. and Lanz T. (eds.)

Mathys G., Lanz T., 1990, A\&A 230, L21

Michaud G., 1970, ApJ 160, 641

Michaud G., Charland Y., Vauclair S., Vauclair G., 1976, ApJ 210,447

Michaud G., Charland Y., 1986, ApJ 311, 326

Moon T.T., Dworetsky M.M., 1985, MNRAS 217, 305

Moon T.T., 1985, Comm. Univ. London Obs. 78

Napiwotzki R., Schönberner D., Wenske V., 1993, A\&A 268, 653

Popper D.M., 1971, ApJ 169, 549

Preston G.W., 1974, ARA\&A 12, 257

Richer J., Michaud G., Turcotte S., 2000, ApJ 529, 338

Roman N., Morgan W.W., Eggen O., 1948, ApJ 107, 107

Schaller G., Schaerer D., Meynet G., Maeder A., 1992, A\&AS 96, 269

Spite M., 1967, Ann. d'Astrophys. 30, 211

Turcotte S., Richer J., Michaud G., 1998, ApJ 504, 559

van't Veer-Menneret C., Mégessier C., 1996, A\&A 309, 879

Wiese W.C., Smith M.W., Miles B.M., 1969, Atom. Trans. Prob., Nat. Stand. Ref. Data Ser. N.B.S. Monograph 22, Washington DC 